\title{
ON A CONJECTURE OF LINDENSTRAUSS AND PERLES IN AT MOST 6 DIMENSIONS
}

\author{
by D. G. LARMAN
}

(Received 18 September, 1976)

1. Introduction. In [1] J. Lindenstrauss and $M$. A. Perles studied the extreme points of the set of all linear operators $T$ of norm $\leqslant 1$ from a finite dimensional Banach space $X$ into itself. In particular they studied the question "When do these extreme points form a semigroup?".

Let $X$ be a Banach space. Then $S(X)$ denotes the unit ball of $X$ and $B(X)$ denotes the unit ball of all operators from $X$ into itself (with the usual operator norm). Let ext $A$ denote the set of extreme points of a set $A$. The two principal theorems of [1] are:

THEOREM 1. The following three assertions, concerning a finite dimensional Banach space $X$, are equivalent:

(1) $x \in \operatorname{ext} S(X), T \in \operatorname{ext} B(X) \Rightarrow T x \in \operatorname{ext} S(X)$;

(2) $T_{1}, T_{2} \in \operatorname{ext} B(X) \Rightarrow T_{1} T_{2} \in \operatorname{ext} B(X)$;

(3) $\left\{T_{i}\right\}_{i=1}^{m} \in \operatorname{ext} B(X) \Rightarrow\left\|T_{1} \ldots T_{m}\right\|=1$, for $m=1,2, \ldots$

THEOREM 2. Let $X$ be a Banach space of dimension $\leqslant 4$. Then $X$ has properties (1) to (3) of Theorem 1 if and only if one of the following conditions holds:

(i) $X$ is an inner product space;

(ii) $S(X)$ is a polytope with the property that for every facet $K$ of $S(X), S(X)$ is the convex hull of $K \cup-K$.

In 5 dimensions they give an example of a polytope $S(X)$ which satisfies (ii) of Theorem 2 but for which $X$ does not have properties (1) to (3) of Theorem 1 . However, they conjecture that any finite dimensional Banach space $X$ which has properties (1) to (3) of Theorem 1 also satisfies (i) or (ii) of Theorem 2. The purpose of this note is to prove this conjecture for Banach spaces $X$ of dimension at most 6 . The methods probably work for higher dimensions but are limited by the large number of cases which need to be considered.

2. Pre-requisites. We state here the definitions and results of [1] which we shall use.

Definition. Let pext $B(X)$ denote the subset of $B(X)$ consisting of all finite products of elements of ext $B(X)$ and let cl pext $B(X)$ denote its closure.

In [1] it was shown that if $X$ satisfies Theorem 1 then

$$
\text { cl pext } B(X)=\operatorname{ext} B(X) \text {. }
$$

Definition. Let $k(X)=\min \{\operatorname{dim} T X: T \in \operatorname{cl}$ pext $B(X)\}$.

Let $X$ be a Banach space of dimension $n$. Then $X$ has properties (1) to (3) of Theorem 1 if and only if $k(X)>0$. If $k(X)=n$ then $X$ is an inner product space, and if

Glasgow Math. J. 19 (1978) 87-97 
$k(X)=1$ then $S(X)$ is a polytope with the property that, for every facet $K$ of $S(X), S(X)$ is the convex hull of $K \cup-K$. The conjecture of Lindenstrauss and Perles therefore is that there does not exist $X$ with $1<k(X)<n$. In [1] it was shown that $k(X) \neq n-1$ or $n-2$ which, of course, proves Theorem 2.

Furthermore it was shown that the following result holds.

Lemma 1. Let $X$ have properties (1) to (3) of Theorem 1 and let $1<k(X)<n$. Say $k(X)=k$. Then ext $S(X)$ is closed and is the union of an infinite number of $k$-dimensional ellipsoids, say ext $S(X)=\cup_{\alpha \in A} X_{\alpha}$ where $X_{\alpha}$ is a $k$-dimensional ellipsoid. Also there is a projection $P_{\alpha}$ in ext $B(X)$ from $X$ to $X_{\alpha}$ and the restriction of every $T \in \operatorname{ext} B(X)$ to $X_{\alpha}$ is an isometry.

Since $k(X)=k\left(X^{*}\right)$, Lemma 1 also holds for $X^{*}$, say ext $S\left(X^{*}\right)$ is the union of an infinite number of $k$-dimensional ellipsoids $\left\{X_{\beta}^{*}\right\}_{\beta \in B}$. The various projections $P_{\alpha}$ induce circumscribing $k$-dimensional elliptic cylinders to $S(X)$, say $\left\{C_{\beta}\right\}_{\beta \in B}$, where $C_{\beta}$ is the polar of $X_{\beta}^{*}, \beta \in B$. Consequently $\left\{C_{\beta}\right\}_{\beta \in B}$ is also infinite and closed in the obvious sense. Also each $X_{\alpha}(\alpha \in A)$ lies on the boundary of each $C_{\beta}(\beta \in B)$.

3. Additional lemmas. If, using the notation of the previous section, we consider a $k$-dimensional ellipsoid $X_{0}$ of the collection $\left\{X_{\alpha}\right\}_{\alpha \in A}$, we may consider $X_{0}$ as a base for $C_{\beta}$ and let $L_{\beta}$ denote the $(n-k)$-subspace of generators of $C_{\beta}$, i.e.

$$
C_{\beta}=X_{0}+L_{\beta}, \beta \in B \text {. }
$$

Then, if $\mathbf{x} \in X_{0},\left(\mathbf{x}+L_{\beta}\right) \cap S(X)$ is a face of $S(X)$ of dimension at most $n-k$. The collection $\left\{C_{\beta}\right\}_{\beta \in B}$ is closed and infinite, and consequently it contains a limit cylinder

$$
C_{\beta_{0}}=X_{0}+L_{\beta_{0}} \text {. }
$$

Our first objective is to establish Lemma 3 which asserts that for each $\mathbf{x} \in X_{0}$, $\left(x+L_{\beta_{n}}\right) \cap S(X)$ has dimension less than $n-k$. To do this, we need to establish

Lemma 2. Let $Y_{m}=\left\{\mathbf{x}:\left(\mathbf{x}-\mathbf{y}_{m}\right)^{t} A_{m}\left(\mathbf{x}-\mathbf{y}_{m}\right) \leqslant \alpha_{m}\right\}\left(\alpha_{m}>0\right)$ be a closed convex elliptic cylinder in $E^{n}$, where $\mathbf{x}^{t} A_{m} \mathbf{x}$ is a positive semi-definite quadratic form, $m=1,2, \ldots$ Suppose that there exist $n+1$ affinely independent points $\mathbf{x}_{1}, \ldots, \mathbf{x}_{n+1}$ which lie on the boundary of each $Y_{m}, m=1,2, \ldots$ Then there exist a subsequence $M$ and a closed convex $n$-dimensional set $Y$ such that

(i) $Y_{m} \cap B \rightarrow Y \cap B$ as $m \rightarrow \infty$ through $M$ for any closed ball $B$,

(ii) $\mathbf{x}_{1}, \ldots, \mathbf{x}_{n+1}$ lie on the boundary of $Y$ and at least one of the line segments $\left[\mathbf{x}_{i}, \mathbf{x}_{j}\right]$ does not lie in the boundary of $Y$.

Proof. By using the Blaschke selection theorem and a standard diagonalisation argument we choose a subsequence $M$ and a closed convex $n$-dimensional set $Y$ such that $Y_{m} \cap B \rightarrow Y \cap B$ as $m \rightarrow \infty$ through $M$ for any closed ball $B$. We suppose that Lemma 2 is false, i.e., that $\left[\mathbf{x}_{i}, \mathbf{x}_{j}\right]$ lies on the boundary of $Y$ for $1 \leqslant i<j \leqslant n+1$. As $\mathbf{x}_{1}, \ldots, \mathbf{x}_{n+1}$ are affinely independent, $\operatorname{conv}\left(x_{1}, \ldots, x_{n+1}\right)$ meets the interior of $Y$. Consequently we may 
pick a $(d+1)$-membered subset $(2 \leqslant d \leqslant n)$, say $\mathbf{x}_{1}, \ldots, \mathbf{x}_{d+1}$, so that $\operatorname{conv}\left(\mathbf{x}_{1}, \ldots, \mathbf{x}_{d+1}\right)$ meets the interior of $Y$ but $\operatorname{conv}\left(x_{i_{1}}, \ldots, \mathbf{x}_{i_{d}}\right)$ is contained in the boundary of $Y$ for $1 \leqslant i_{1}<\ldots<i_{d} \leqslant d+1$.

Let $D$ be the affine space spanned by $x_{1}, \ldots, x_{d+1}$. Then $D \cap Y_{m} \rightarrow \operatorname{conv}\left(x_{1}, \ldots, x_{d+1}\right)$ as $m \rightarrow \infty$ in $M$. Consequently $D \cap Y_{m}$ is bounded for sufficiently large $m$ in $M$, and so $D \cap Y_{m}$ is a $d$-dimensional ellipsoid for sufficiently large $m$ in $M$. But then $D \cap Y_{m}$ is centrally symmetric and so $\operatorname{conv}\left(\mathbf{x}_{1}, \ldots, \mathbf{x}_{d+1}\right)$ is centrally symmetric, which is not so. This contradiction establishes Lemma 2.

Lemma 3. The subset $X_{0}^{n-k}=\left\{\mathbf{x}:\left(\mathbf{x}+L_{\beta_{0}}\right) \cap S(X)\right.$ has dimension $\left.n-k\right\}$ of $X_{0}$ is empty.

Proof. We suppose that the lemma is false. Let $y_{0} \in X_{0}^{n-k}$. Then $\left(y_{0}+L_{\beta_{0}}\right) \cap S(X)$ contains $n-k+1$ affinely independent extreme points $\mathbf{y}_{0}, \ldots, \mathbf{y}_{n-k}$. Each of these extreme points $\mathbf{y}_{0}, \ldots, \mathbf{y}_{n-k}$ is contained in (at least) one $k$-dimensional ellipsoid, $X_{0}, \ldots, X_{n-k}$ respectively say, from amongst the collection $\left\{X_{\alpha}\right\}_{\alpha \in A}$.

Now $C_{\beta_{0}}=X_{0}+L_{\beta_{0}}$ is a limit cylinder of the collection $\left\{C_{\beta}\right\}_{\beta \in B}$, and so we can choose distinct cylinders

$$
C_{\beta_{m}}=X_{0}+L_{\beta_{m}}, \quad m=0,1,2, \ldots
$$

so that $C_{\beta_{m}} \rightarrow C_{\beta_{0}}$ as $m \rightarrow \infty$. The set $Y_{m}=\left(y_{0}+L_{\beta_{0}}\right) \cap\left(X_{0}+L_{\beta_{m}}\right)$ is the intersection of the flat $\mathbf{y}_{0}+L_{\beta_{0}}$ with the elliptic cylinder $X_{0}+L_{\beta_{m}}$ and consequently $Y_{m}$ is also an elliptic cylinder (possibly an ellipsoid) in $\mathbf{y}_{0}+L_{\beta_{0}}$.

By Lemma 2 , there exist a subsequence $M$ and a closed convex $(n-k)$-dimensional set $Y$ in $y_{0}+L_{\beta_{0}}$ so that

(i) $Y_{m} \cap B \rightarrow Y \cap B$ as $m \rightarrow \infty$ through $M$ for any closed ball $B$ in $y_{0}+L_{\beta_{0}}$,

(ii) $y_{0}, \ldots, y_{n-k}$ lie on the relative boundary of $Y$ and at least one of the line segments $\left[\mathbf{y}_{i}, \mathbf{y}_{j}\right]$ does not lie in the boundary of $Y$.

We may suppose, without loss of generality, that $\left[\mathbf{y}_{0}, \mathbf{y}_{1}\right]$ does not lie in the relative boundary of $Y$. Now, by continuity there exists a neighbourhood $U$ of $y_{0}$ in $X_{0}$ such that $U$ is contained in $X_{0}^{n-k}$. Let $x_{m}$ be that point of $X_{0}$ such that $y_{1}$ and $x_{m}$ lie on the same face of $C_{m}$, i.e., $\mathbf{x}_{m}=\left(\mathbf{y}_{1}+L_{\boldsymbol{\beta}_{m}}\right) \cap X_{0}$. Then, since $C_{\beta_{m}} \rightarrow C_{\beta_{0}}$ as $m \rightarrow \infty, \mathbf{x}_{m} \rightarrow \mathbf{y}_{0}$ as $m \rightarrow \infty$. Also, if $\mathbf{x}_{m}=\mathbf{y}_{0}$ for all but finitely many $m \in M$ then the line segment $\left[\mathbf{y}_{1}, \mathbf{y}_{0}\right]$ lies on the same face of $C_{\beta m} \cap\left(y_{0}+L_{\beta_{0}}\right)=Y_{m}$ for all but finitely many $m \in M$. So $\left[y_{1}, y_{0}\right]$ is on the boundary of $Y$, which yields a contradiction. Consequently, we may suppose that $\mathbf{x}_{m} \neq \mathbf{y}_{0}$ for all $m \in M$.

There will be a hyperplane of support, say $H_{m}$, to $X_{0}+L_{\beta_{m}}$, and hence to $S(X)$, which contains both $\mathbf{x}_{m}$ and $\mathbf{y}_{1}$. Then $\left(\mathbf{y}_{0}+L_{\beta_{0}}\right) \cap H_{m}$ is a hyperplane in $\mathbf{y}_{0}+L_{\beta_{0}}$ which supports $\left(\mathbf{y}_{0}+L_{\beta_{0}}\right) \cap S(X)$ at $\mathbf{y}_{1}$. Since $\left[\mathbf{y}_{0}, \mathbf{y}_{1}\right]$ does not lie in the relative boundary of $Y,\left(\mathbf{y}_{0}+L_{\beta_{0}}\right) \cap$ $H_{m}$ may be supposed to converge to a hyperplane $\left(\mathbf{y}_{0}+L_{\beta_{0}}\right) \cap H$, and $H_{m}$ converges to $H$, which supports $Y$ at $\mathbf{y}_{1}$ and $\mathbf{y}_{0} \notin\left(\mathbf{y}_{0}+L_{\beta_{0}}\right) \cap H$.

Now consider a line segment $\left[\mathbf{y}_{0}, \mathbf{z}_{1}\right]$ passing through the relative interior of $\mathbf{y}_{0}+$ $\left.L_{\beta_{0}}\right) \cap S(X)$, where $\mathbf{z}_{1} \in \operatorname{relbdy}\left\{\left(y_{0}+L_{\beta_{0}}\right) \cap S(X)\right\}$ and $z_{1}$ is chosen so close to $\mathbf{y}_{1}$ as to ensure that the hyperplane $H$ cuts the line through $\left[\mathbf{z}_{1}, \mathbf{y}_{0}\right]$ in a point $\mathbf{b}_{1}$, where $\mathbf{b}_{1}, \mathbf{z}_{1}, \mathbf{y}_{0}$ occur in that order. Consequently, we may suppose that for $m \in M, H_{m}$ cuts the line through $\left[\mathbf{z}_{1}, \mathbf{y}_{0}\right]$ in a point $\mathbf{b}_{m}$, where $\mathbf{b}_{m}, \mathbf{z}_{1}, \mathbf{y}_{0}$ occur in that order. 
Consider next the 3-dimensional subspace $G_{m}$ generated by $\mathbf{z}_{1}, \mathbf{y}_{0}$ and $\mathbf{x}_{m}$. In $G_{m}$ the 2-plane $H_{m} \cap G_{m}$ contains $\mathbf{b}_{m}$ and $\mathbf{x}_{m}$ and is tangent to $X_{0} \cap G_{m}$. Consequently $H_{m} \cap G_{m}$ strictly separates the point $\mathbf{y}_{0}$ from the ray

$$
\ell_{m}=\left\{\mathbf{x}_{m}+t\left(\mathbf{z}_{1}-\mathbf{y}_{0}\right), t>0\right\}
$$

So $\ell_{m}$ does not meet the face $\left(\mathbf{x}_{m}+L_{\beta_{0}}\right) \cap S(X)$. But

$$
\ell_{0}=\lim _{m \rightarrow \infty} \ell_{m}=\left\{\mathbf{y}_{0}+t\left(\mathbf{z}_{1}-\mathbf{y}_{0}\right), t>0\right\}
$$

meets $\left(\mathbf{y}_{0}+L_{\beta_{0}}\right) \cap S(X)$ in a relatively interior point $\mathbf{z}=\left(\mathbf{z}_{1}+\mathbf{y}_{0}\right) / 2$. So there exists a finite set $\mathbf{q}_{1}, \ldots, \mathbf{q}_{\mathrm{p}}$ of extreme points of $S(X)$ in $\left(\mathrm{y}_{0}+L_{\beta_{0}}\right) \cap S(X)$ whose convex hull is $(n-k)$-dimensional and contains $\mathbf{z}$ as a relatively interior point. Let $X_{\alpha_{1}}, \ldots, X_{\alpha_{p}}$ be ellipsoids amongst $\left\{X_{\alpha}\right\}_{\alpha \in A}$ such that $\mathbf{q}_{i} \in X_{\alpha_{i}}(i=1, \ldots, p)$, and let $q_{i}^{m}=X_{\alpha_{i}} \cap\left(\mathbf{x}_{m}+L_{\beta_{0}}\right)$ $(i=1, \ldots, p)$. Then $\mathbf{q}_{i}^{m_{m}} \rightarrow \mathbf{q}_{i}$ as $m \rightarrow \infty(i=1, \ldots, p)$ and so, for sufficiently large $m$ in $M$, $\mathbf{z}^{m}=\mathbf{x}_{m}+\left(\mathbf{z}_{1}-\mathbf{y}_{0}\right) / 2$ lies in the relative interior of the $(n-k)$-dimensional set $\operatorname{conv}\left(\mathbf{q}_{1}^{m}, \ldots, \mathbf{q}_{p}^{m}\right)$ which is contained in $\left(\mathbf{x}_{m}+L_{\beta_{0}}\right) \cap S(X)$. This contradicts the previous result that $\ell_{m}$ does not meet $\left(\mathrm{x}_{m}+L_{\beta_{0}}\right) \cap S(X)$ and completes the proof of Lemma 3. [1].

The next lemma uses an extension of the methods used to prove Proposition 4.3 of

Lemma 4. Let $X$ be a finite dimensional Banach space with $0<k(X)<n$. If $[\mathbf{a}, \mathbf{b}]$ is an edge of $S(X)$ then there must be at least 2 members of $\left\{X_{\alpha}\right\}_{\alpha \in A}$ which contain $\mathbf{b}$.

Proof. If the lemma is false, then there is an edge $[\mathbf{a}, \mathbf{b}]$ of $S(X)$ such that $\mathbf{b}$ is contained in exactly one member $X_{1}$ of $\left\{X_{\alpha}\right\}_{\alpha \in A}$. Let $Z$ be the 2-dimensional subspace of $X$ spanned by $[\mathbf{a}, \mathbf{b}]$ and let $k=k(X)$. Then, if $B\left(E^{k}, X\right)$ denotes the set of linear operators of norm at most 1 from $E^{k}$ to $X$, there exists $T \in B\left(E^{k}, X\right)$ such that

$$
T \mathbf{e}_{1}=\frac{1}{2}(\mathbf{a}+\mathbf{b}), T\left(\alpha \mathbf{e}_{1}+\beta \mathbf{e}_{2}\right)=\mathbf{y} \in Z
$$

with $\alpha^{2}+\beta^{2}=\|\mathbf{y}\|=1, \beta \neq 0$ and $T \mathbf{e}_{i}=0$ for $i>2$ (here $\left\{\mathbf{e}_{i}\right\}_{i=1}^{k}$ denotes the usual coordinate basis of $\left.E^{k}\right)$. Let $T=\sum_{i=1}^{q} \lambda_{i} T_{i}$ with $\lambda_{i}>0(i=1, \ldots, q), \sum_{i=1}^{q} \lambda_{i}=1$ and $T_{i} \in \operatorname{ext} B\left(E^{k}, X\right)$ $(i=1, \ldots, q)$. Then, since $T_{i}$ takes extreme points to extreme points (see Lemmas 3.11-13 of [1]), $T_{i} \mathbf{e}_{1}=\mathbf{a}$ or $\mathbf{b}$ for $i=1, \ldots, q$. We assume that $T_{i} \mathbf{e}_{1}=\mathbf{a}$ for $i=1, \ldots, p$ and $T_{i} \mathbf{e}_{1}=\mathbf{b}$ for $i=p+1, \ldots, q$. Then we have $1 / 2=\sum_{i=1}^{p} \lambda_{i}=\sum_{i=p+1}^{q} \lambda_{i}$, and $T_{i}$ is an isometry from $E^{k}$ to $X_{1}$ for $i=p+1, \ldots, q$. Let

and

$$
\mathbf{y}_{0}=2 \sum_{i=1}^{p} \lambda_{i} T_{i}\left(\alpha \mathbf{e}_{1}+\beta \mathbf{e}_{2}\right)
$$

$$
\mathbf{y}_{1}=2 \sum_{i=p+1}^{q} \lambda_{i} T_{i}\left(\alpha \mathbf{e}_{1}+\beta \mathbf{e}_{2}\right)
$$


Then $y=\left(y_{0}+y_{1}\right) / 2$, and consequently $y_{0}$ and $y_{1}$ lie on the boundary of $S(X)$. Also $y_{1}$ lies on the relative boundary of $X_{1}$ and so

$$
T_{i}\left(\alpha \mathbf{e}_{1}+\beta \mathbf{e}_{2}\right)=\mathbf{y}_{1}, \quad p+1 \leqslant i \leqslant q .
$$

Since $\beta \neq 0, \mathbf{y}_{1} \neq \pm \mathbf{b}$.

Since $X_{1}$ meets the subspace spanned by $\mathbf{a}, \mathbf{b}$ only at $\pm \mathbf{b}$, it follows that $\mathbf{a}, \mathbf{b}, \mathbf{y}_{0}, \mathbf{y}_{1}$ span a 3-dimensional subspace $F$. Using the methods of Lemma 3.8 of [1], we see that there exists $V \in$ ext $B(X)$ such that $V(\mathbf{a})=V(\mathbf{b})=\mathbf{b}$. Consequently, if $M$ denotes the $(k+1)$-dimensional subspace generated by $X_{1}$ and $\mathbf{b}-\mathbf{a}, V(S(X) \cap M)=X_{1}$. So the cylinder

$$
C=\left\{\left(F \cap X_{1}\right)+t(\mathbf{b}-\mathbf{a}), t \text { real }\right\}
$$

supports $F \cap S(X)$ and contains $F \cap X_{1}$ on its boundary; further, [a, b] is contained in a generator of $C$.

Similarly, considering $\left[\mathbf{y}_{0}, \mathbf{y}_{1}\right]$ and $W \in \operatorname{ext} B(X)$ with $W\left(\mathbf{y}_{0}\right)=W\left(\mathbf{y}_{1}\right)=\mathbf{b}$, we see that there exists a cylinder

$$
C^{\prime}=\left\{\left(F \cap X_{1}\right)+t\left(\mathbf{y}_{1}-\mathbf{y}_{0}\right), t \text { real }\right\}
$$

which supports $F \cap S(X)$ and contains $F \cap X_{1}$ on its boundary; also $\left[\mathbf{y}_{0}, \mathbf{y}_{1}\right]$ is contained in one of the generators of $C^{\prime}$. Since $0 \in \operatorname{lin}\left(\mathbf{a}, \mathbf{b},\left(\mathbf{y}_{0}+\mathbf{y}_{1}\right) / 2\right), \mathbf{y}_{1}-\mathbf{y}_{0}$ is parallel to $\mathbf{b}-\mathbf{a}$ only if $\mathbf{y}_{1}= \pm \mathbf{b}$, which is impossible. So $C^{\prime}$ is not $C$ and again $\mathbf{0} \in \operatorname{lin}\left(\mathbf{a}, \mathbf{b},\left(\mathbf{y}_{0}+\mathbf{y}_{1}\right) / 2\right)$ only if $\mathbf{y}_{1}$ is $\pm \mathbf{b}$, which is impossible. This establishes Lemma 4.

LeMma 5. Let $C$ be a convex body in $E^{n}$ such that ext $C$ is contained in $L_{1} \cup L_{2}$, where $L_{1}$ and $L_{2}$ are hyperplanes. Then, if $\mathbf{y}$ belongs to (ext $\left.C\right) \cap\left(L_{1} \backslash L_{2}\right)$, there is an edge of $C$ which contains $\mathbf{y}$.

Proof. The result is trivial when $n=2$ and, proceeding by induction, it is enough to find a proper face $F$ of $C$ which contains $\mathbf{y}$ but which is not contained in $L_{1}$.

Let $H$ be a hyperplane of support to $C$ at $\mathbf{y}$. If $L_{1} \cap L_{2} \neq \varnothing$, we may suppose, by taking a projective transformation if necessary, that $H \cap L_{1}$ contains a translate of $L_{2} \cap L_{1}$. Then, if $\Pi$ denotes the orthogonal projection of $E^{n}$ along $L_{1} \cap L_{2}, y$ is an extreme point of the 2-dimensional convex body $\Pi C$. The point $\Pi y$ is not in $\Pi L_{2}$ and ext $\Pi C$ is contained in $\Pi L_{1} \cup \Pi L_{2}$. So there exists an edge $F^{*}$ of $\Pi C$ which contains $\Pi y$ but which is not contained in $\Pi L_{1}$. Then $F=C \cap \Pi^{-1} F^{*}$ is the required face of $C$.

If $L_{1} \cap L_{2}=\varnothing$ i.e., $L_{1}$ is parallel to $L_{2}$, then it is possible to choose $H$ so that $H \neq L_{1}$. Then we project along $H \cap L_{1}$ and argue as before.

Lemma 6. Let $X$ be a 6-dimensional Banach space with $k(X)=2$. Then there are no points on $S(X)$ which lie on two distinct members of $\left\{X_{\alpha}\right\}_{\alpha \in A}$. Consequently $S(X)$ does not contain any edges.

Proof. We suppose that the lemma is false. Let $X_{1}, X_{2}$ be two ellipses of $\left\{X_{\alpha}\right\}_{\alpha \in A}$ which intersect. Without loss of generality we may suppose that

$$
X_{1}: x_{1}^{2}+x_{2}^{2}=1, \quad x_{3}=x_{4}=x_{5}=x_{6}=0,
$$


and

$$
X_{2}: x_{2}^{2}+x_{3}^{2}=1, \quad x_{1}=x_{4}=x_{5}=x_{6}=0,
$$

which intersect in the point $(0,1,0,0,0,0)^{\prime}$. Then the cylinders $\left\{C_{\beta}\right\}_{\beta \in B}$ which arise from the dual ellipses $\left\{X_{\beta}^{*}\right\}_{\beta \in B}$ of $S\left(X^{*}\right)$ meet the 3-dimensional space $x_{4}=x_{5}=x_{6}=0$ in cylinders of the form

$$
x_{2}^{2}+\left(x_{1} \pm x_{3}\right)^{2}=1 \text {, }
$$

and hence their generators contain one of $(1,0, \pm 1,0,0,0)^{\prime}$. This means that each of the ellipses $X_{\beta}^{*}$ is orthogonal to one of $(1,0, \pm 1,0,0,0)^{\prime}$ and hence the extreme points of $S\left(X^{*}\right)$ are contained in two 5-dimensional subspaces $L_{1}^{*}, L_{2}^{*}$. Consequently, if $X_{1}^{*}$ is one of the collection $\left\{X_{\beta}^{*}\right\}_{\beta \in B}$ such that $X_{1}^{*} \cap\left(L_{1}^{*} \backslash L_{2}^{*}\right) \neq \varnothing$, then, using Lemma 5 , if $\mathbf{y}^{*} \in$ $X_{1}^{*} \cap\left(L_{1}^{*} \backslash L_{2}^{*}\right)$ there exists an edge of $S\left(X^{*}\right)$ which contains $\mathbf{y}^{*}$. So, using Lemma 4 , there exists an ellipse of $\left\{X_{\beta}^{*}\right\}_{\beta \in B}$, different from $X_{1}^{*}$, which contains $\mathbf{y}^{*}$.

Let $\mathbf{y}_{2}^{*}, \mathbf{y}_{3}^{*}$ be distinct points of $X_{1}^{*} \cap\left(L_{1} \backslash L_{2}\right)$ and let $X_{2}^{*}, X_{3}^{*}$ be distinct from $X_{1}^{*}$ and contain $\mathbf{y}_{2}^{*}, \mathbf{y}_{3}^{*}$ respectively. We now disregard the special forms, assumed previously, for $X_{1}$ and $X_{2}$ and we may instead assume that

$$
\begin{array}{ll}
X_{1}^{*}: x_{1}^{2}+x_{2}^{2}=1, & x_{3}=x_{4}=x_{5}=x_{6}=0, \\
X_{2}^{*}: x_{2}^{2}+x_{3}^{2}=1, & x_{1}=x_{4}=x_{5}=x_{6}=0, \\
X_{3}^{*}: x_{1}^{2}+x_{4}^{2}=1, & x_{2}=x_{3}=x_{5}=x_{6}=0,
\end{array}
$$

and hence that

$$
\begin{aligned}
& \mathbf{y}_{2}^{*}=(0,1,0,0,0,0)^{\prime}, \\
& \mathbf{y}_{3}^{*}=(1,0,0,0,0,0)^{\prime},
\end{aligned}
$$

Then each cylinder arising from the ellipses in $\left\{X_{\alpha}\right\}_{\alpha \in A}$ meets the 4-dimensional subspace $x_{5}=x_{6}=0$ in a cylinder of the form

$$
\left(x_{1} \pm x_{3}\right)^{2}+\left(x_{2} \pm x_{4}\right)^{2}=1 \text {. }
$$

So each cylinder arising from $\left\{X_{\alpha}\right\}_{\alpha \in A}$ contains amongst its generators one of the four 2-dimensional subspaces

$$
x_{1}= \pm x_{3}, \quad x_{2}= \pm x_{4}, \quad x_{5}=x_{6}=0,
$$

and not all of these cylinders can share a common generator. This means that the extreme points of $S(X)$ are contained in at least two and at most four 4-dimensional subspaces $L_{i_{1}}, \ldots, L_{i_{1}}$ and $L_{i_{1}} \cap \ldots \cap L_{i_{1}}$ is the 2-dimensional subspace $L: x_{1}=x_{2}=x_{3}=x_{4}=0$. We may suppose that

$$
(\operatorname{ext} S(X)) \backslash \bigcup_{\substack{i=1 \\ i \neq k}}^{j} L_{\mathrm{i}} \neq \varnothing(k=1, \ldots, j),
$$

for otherwise $L_{k}$ is redundant. For each $L_{i_{k}}$ we may pick $X_{1}, X_{2}, X_{3}$ as $X_{1}^{*}, X_{2}^{*}, X_{3}^{*}$ were chosen above, and we deduce that the cylinders arising from $\left\{X_{\beta}^{*}\right\}_{\beta \in B}$ contain, amongst 
their generators, one of four 2-dimensional subspaces $L_{i, 1}, \ldots, L_{i, 4}$, at most one of which can be $L$ and all of which lie in $L_{i k}$.

We may classify the cylinders arising from the $\left\{X_{\beta}^{*}\right\}_{\beta \in B}$ into a finite number of classes according to which of the 2-dimensional spaces $L_{i_{k}, 1}, \ldots, L_{i_{k}, 4}$ are contained amongst its generators $(k=1, \ldots, j)$. It is only in the class (if it exists) in which $L$ occurs as the 2-dimensional subspace for each $k$ that a 3-dimensional subspace of generators is not determined.

In $S\left(X^{*}\right)$, this means that the extreme points of $S\left(X^{*}\right)$ are contained in finitely many 3-dimensional subspaces $M_{1}, \ldots, M_{\mathrm{p}}$ and at most one 4-dimensional subspace $N$. Now $M_{1}, \ldots, M_{\mathrm{p}}$ can contain at most two members each of $\left\{X_{\beta}^{*}\right\}_{\beta \in B}$, and so there are only finitely many $X_{\beta}^{*}$ that are not wholly contained in $N$.

There are two 5-dimensional subspaces $N_{1}, N_{2}$ which contain ext $S\left(X^{*}\right)$ and we may suppose that $(\operatorname{ext} S(X)) \cap\left(N_{1} \backslash N\right) \neq \varnothing$ and hence is infinite. By Lemmas 4, 5 it follows that for each point $y \in($ ext $S(X)) \cap\left(N_{1} \backslash N\right)$ there are at least two members of $\left\{X_{\beta}^{*}\right\}_{\beta \in B}$ which contain y. Consequently, there are infinitely many $\left\{X_{\beta}^{*}\right\}_{\beta \in B}$ which are not contained in $N$. This contradiction establishes Lemma 6.

Lemma 7. Let $X$ be a 5- or 6-dimensional Banach space. Then $k(X) \neq 2$.

Proof. We only prove the lemma in the harder 6-dimensional case. We choose $C_{\beta_{0}}=X_{0}+L_{\beta_{0}}$ as in Lemma 3 with $k(X)=2$, and deduce that the subset

$$
X_{0}^{4}=\left\{\mathbf{x}:\left(\mathbf{x}+L_{\beta_{0}}\right) \cap S(X) \text { has dimension } 4\right\}
$$

of $X_{0}$ is empty.

If $\left(\mathbf{x}+L_{\beta_{0}}\right) \cap S(X)$ has dimension 3 , let $H$ be the affine hull of $\left(\mathbf{x}+L_{\beta_{0}}\right) \cap S(X)$. Any cylinder $C_{\beta}=X_{0}+L_{\beta}$, with $L_{\beta} \neq L_{\beta_{0}}$, meets $H$ in a cylinder $H \cap C_{\beta}$ which is either the product of an ellipse and a line or the product of a line segment and a plane. The extreme points of $\left(\mathbf{x}+L_{\beta_{0}}\right) \cap S(X)$ must lie on the relative boundary of $H \cap C_{\beta}$ and so $\left(x+L_{\beta_{0}}\right) \cap$ $S(X)$ must contain edges of $S(X)$, which contradicts Lemma 6 .

So, $\left(x+L_{\beta_{0}}\right) \cap S(X)$ is either the single point $\mathbf{x}$ or a 2-dimensional ellipse, for each $\mathbf{x} \in X_{0}$. Since $\left\{X_{\alpha}\right\}_{\alpha \in A}$ is infinite, $\left(x+L_{\beta_{0}}\right) \cap S(X)$ is an ellipse, except for possibly two opposite points of $X_{0}$.

Consider next a sequence of distinct cylinders $C_{\beta_{m}}=X_{0}+L_{\beta_{m}}(m=0,1,2, \ldots)$, which converge to $C_{\beta_{0}}$ as $m \rightarrow \infty$, and an ellipse $E=\left(\mathbf{x}+L_{\beta_{0}}\right) \cap S(X)$. Unless $\mathbf{x}+L_{\beta_{m}}$ contains $E$, the projection of $E$ along $L_{\beta_{m}}$, into $X_{0}$, must be an ellipse on $X_{0}$ and so must coincide with $X_{0}$. But, as $m \rightarrow \infty$, this projection must converge to $\mathbf{x}$, which would be impossible. So we conclude that there exists $M(\mathbf{x})$, such that if $m \geqslant M(\mathbf{x}), \mathbf{x}+L_{\beta_{m}}$ contains $E$. So $L_{\beta_{m}}$ contains the 2-dimensional subspace $D(x)=\operatorname{lin}\{E-x\}$. As $S(X)$ is 6-dimensional and $X_{0}$ is only 2-dimensional, we must be able to choose $\mathbf{x}_{1}, \mathbf{x}_{2}, \mathbf{x}_{3}$ in $X_{0}$ such that $D\left(\mathbf{x}_{1}\right), D\left(\mathbf{x}_{2}\right), D\left(\mathbf{x}_{3}\right)$ arise from ellipses $\left(\mathbf{x}_{i}+L_{\beta_{0}}\right) \cap S(X)(i=1,2,3)$ and span the 4-dimensional subspace $L_{\beta_{0}}$. Then, if $m \geqslant \max _{1<i<3} M\left(x_{i}\right), L_{\beta_{m}}=L_{\beta_{0}}$ and so $C_{\beta_{m}}=C_{\beta_{0}}$, which contradicts the fact that the cylinders $\left\{C_{\boldsymbol{B}_{m}}\right\}_{m=0}^{\infty}$ are distinct. 
Lemma 8. Let $X$ be a 6-dimensional Banach space. Then $k(X) \neq 3$.

Proof. We suppose that $k(X)=3$. Then, using Lemma $3,\left(x+L_{\beta_{0}}\right) \cap S(X)$ is at most 2-dimensional for all $x \in X_{0}$. If two ellipses do not coincide then they meet in at most four points. So, if $\left(x+L_{\beta_{0}}\right) \cap S(X)$ is not an ellipse then it is either a single point, an edge or a 2-dimensional convex set whose boundary consists of at most four edges. Hence, as $\left\{X_{\alpha}\right\}_{\alpha \in A}$ is infinite, for almost all $\mathbf{x}$ in $X_{0},\left(x+L_{\beta_{0}}\right) \cap S(X)$ is a 2-dimensional ellipse.

We may suppose that $X_{0}$ is the 3 -sphere

$$
x_{1}^{2}+x_{2}^{2}+x_{3}^{2}=1, \quad x_{4}=x_{5}=x_{6}=0
$$

and that one of these ellipses $\left(x+L_{\beta_{0}}\right) \cap S(X)$ is

$$
\left(x_{4}-1\right)^{2}+x_{5}^{2}=1, \quad x_{1}=1, \quad x_{2}=x_{6}=0,
$$

where $\mathbf{x}=(1,0,0,0,0,0)^{\prime}$.

Consider any 3-cylinder arising from $\left\{X_{\beta}^{*}\right\}_{\beta \in B}$ intersected with the 5-dimensional subspace $x_{6}=0$. This has equation

$$
\left(x_{1}+\alpha_{1} x_{4}+\beta_{1} x_{5}\right)^{2}+\left(x_{2}+\alpha_{2} x_{4}+\beta_{2} x_{5}\right)^{2}+\left(x_{3}+\alpha_{3} x_{4}+\beta_{3} x_{5}\right)^{2}=1 .
$$

If we consider the subset lying in the 2-dimensional affine subspace

$$
x_{1}=1 . \quad x_{2}=x_{3}=x_{6}=0,
$$

we obtain

$$
\left(1+\alpha_{1} x_{4}+\beta_{1} x_{5}\right)^{2}+\left(\alpha_{2} x_{4}+\beta_{2} x_{5}\right)^{2}+\left(\alpha_{3} x_{4}+\beta_{3} x_{5}\right)^{2}=1,
$$

which must be equivalent to

$$
\left(x_{4}-1\right)^{2}+x_{5}^{2}=1 .
$$

So $\alpha_{1}=-1, \beta_{1}=0, \alpha_{2}=\alpha_{3}=0, \beta_{2}^{2}+\beta_{3}^{2}=1$. Hence if we write $\beta_{2}=\cos \lambda, \beta_{3}=\sin \lambda$ the 3 -cylinder, intersected with $x_{6}=0$, then has the form

$$
\left(x_{1}-x_{4}\right)^{2}+\left(x_{2}+x_{5} \cos \lambda\right)^{2}+\left(x_{3}+x_{5} \sin \lambda\right)^{2}=1 \text {, }
$$

or

$$
x_{1}^{2}+x_{2}^{2}+x_{3}^{2}+x_{4}^{2}+x_{5}^{2}-1-2 x_{1} x_{4}=-2 x_{5}\left(x_{2} \cos \lambda+x_{3} \sin \lambda\right) \text {. }
$$

If there is an extreme point of $S(X)$ in the 5-dimensional subspace $x_{6}=0$ which does not lie in either $x_{5}=0$ or $x_{2}=x_{3}=0$, then $\lambda$ can take one of two values $\lambda_{1}, \lambda_{2}$ in $[0,2 \pi]$. Say

with

$$
\mathbf{y}=\left(y_{1}, y_{2}, y_{3}, y_{4}, y_{5}, y_{6}\right)^{\prime}
$$

$$
y_{1}^{2}+y_{2}^{2}+y_{3}^{2}+y_{4}^{2}+y_{5}^{2}-1-2 y_{1} y_{4}=-2 y_{5}\left(y_{2} \cos \lambda+y_{3} \sin \lambda\right) \text {. }
$$

Then the two sets of 2-dimensional generators for the cylinders are given by

and

$$
x_{1}=x_{4}, \quad x_{2}=-x_{5} \cos \lambda_{1}, \quad x_{3}=-x_{5} \sin \lambda_{1}
$$

$$
x_{1}=x_{4}, \quad x_{2}=-x_{5} \cos \lambda_{2}, \quad x_{3}=-x_{5} \sin \lambda_{2} \text {. }
$$


So both sets of generators lie in the 3 -space

$$
x_{1}=x_{4}, \quad x_{2} y_{2}+x_{3} y_{3}=c x_{5},
$$

where $c$ is a constant determined by $\mathbf{y}$. Hence the two sets of generators intersect, i.e., all the cylinders $\left\{C_{\beta}\right\}_{\beta \in B}$ have a common generator, which is impossible.

So any point of ext $S(X)$ in $x_{6}=0$ must lie in either the set $x_{5}=0$, or in $x_{2}=x_{3}=0$, or in both. Each of the 3-spheres meets $x_{6}=0$ in at least a 2 -sphere. If one of these 3 -spheres $X_{\gamma}$, other than $X_{0}$, meets $x_{5}=0, x_{6}=0$ in a 2 -sphere, then $X_{0}$ and $X_{\gamma}$ intersect. Otherwise, any two 3-spheres of $\left\{X_{\alpha}\right\}_{\alpha \in A}$ meet the 3-dimensional subspace $x_{2}=x_{3}=x_{6}=$ 0 in at least a 2 -sphere and so intersect. So we may suppose, in any event, that there are two 3 -spheres $X_{1}, X_{2}$ of the collection $\left\{X_{\alpha}\right\}_{\alpha \in A}$ which intersect. If $X_{1}$ is

$$
x_{1}^{2}+x_{2}^{2}+x_{3}^{2}=1, \quad x_{4}=x_{5}=x_{6}=0,
$$

then we may suppose that the other 3 -sphere $X_{2}$ is one of

$$
\begin{array}{ll}
\text { (i) } x_{2}^{2}+x_{3}^{2}+x_{4}^{2}=1, & x_{1}=x_{5}=x_{6}=0, \\
\text { (ii) } x_{3}^{2}+x_{4}^{2}+x_{5}^{2}=1, & x_{1}=x_{2}=x_{6}=0 .
\end{array}
$$

Consider first case (i). Any cylinder arising from $\left\{X_{\beta}^{*}\right\}_{\beta \in B}$ meets the 4-dimensional subspace $x_{5}=x_{6}=0$ in a cylinder of the form

$$
\left(x_{1}+\alpha_{1} x_{4}\right)^{2}+\left(x_{2}+\alpha_{2} x_{4}\right)^{2}+\left(x_{3}+\alpha_{3} x_{4}\right)^{2}=1 .
$$

In the 3-dimensional subspace $x_{1}=x_{5}=x_{6}=0$, this reduces to

$$
\alpha_{1}^{2} x_{4}^{2}+\left(x_{2}+\alpha_{2} x_{4}\right)^{2}+\left(x_{3}+\alpha_{3} x_{4}\right)^{2}=1,
$$

which must be equivalent to

$$
x_{2}^{2}+x_{3}^{2}+x_{4}^{2}=1
$$

So $\alpha_{1}= \pm 1, \alpha_{2}=\alpha_{3}=0$, i.e., all the cylinders have one of $( \pm 1,0,0,0,0,0)^{\prime}$ amongst their generators. Dually, this means that the extreme points of $S\left(X^{*}\right)$ are contained in two 5-dimensional subspaces $L_{1}$ and $L_{2}$. So the cylinders arising from $\left\{X_{\alpha}\right\}_{\alpha \in A}$ give rise to faces of $S\left(X^{*}\right)$ whose extreme points are (almost always) disconnected. So these faces cannot (almost always) be ellipses, which gives the required contradiction in case (i).

Consider next $X_{2}$ as in (ii). Any cylinder arising from $\left\{X_{\beta}^{*}\right\}_{\beta \in B}$ meets $x_{6}=0$ in a cylinder of the form

$$
\left(x_{1}+\alpha_{1} x_{4}+\beta_{1} x_{5}\right)^{2}+\left(x_{2}+\alpha_{2} x_{4}+\beta_{2} x_{5}\right)^{2}+\left(x_{3}+\alpha_{3} x_{4}+\beta_{3} x_{5}\right)^{2}=1,
$$

which, when also $x_{1}=x_{2}=0$, has the form

$$
\left(\alpha_{1} x_{4}+\beta_{1} x_{5}\right)^{2}+\left(\alpha_{2} x_{4}+\beta_{2} x_{5}\right)^{2}+\left(x_{3}+\alpha_{3} x_{4}+\beta_{3} x_{5}\right)^{2}=1,
$$

which must be

$$
x_{3}^{2}+x_{4}^{2}+x_{5}^{2}=1
$$


Consequently,

$$
\alpha_{3}=\beta_{3}=0, \quad \alpha_{1}^{2}+\alpha_{2}^{2}=1, \quad \beta_{1}^{2}+\beta_{2}^{2}=1, \quad \alpha_{1} \beta_{1}+\alpha_{2} \beta_{2}=0 .
$$

Let $\alpha_{1}=\cos \lambda, \alpha_{2}=\sin \lambda, \beta_{1}=\cos \rho, \beta_{2}=\sin \rho$. Then

that is,

$$
\cos \lambda \cos \rho+\sin \lambda \sin \rho=0,
$$

$$
\cos (\lambda-\rho)=0 .
$$

So $\rho=\lambda+3 \pi / 2$ or $\rho=\lambda+\pi / 2$. Hence the cylinder has the form

or

$$
\left(x_{1}+x_{4} \cos \lambda+x_{5} \sin \lambda\right)^{2}+\left(x_{2}+x_{4} \sin \lambda-x_{5} \cos \lambda\right)^{2}+x_{3}^{2}=1,
$$

$$
\left(x_{1}+x_{4} \cos \lambda-x_{5} \sin \lambda\right)^{2}+\left(x_{2}+x_{4} \sin \lambda+x_{5} \cos \lambda\right)^{2}+x_{3}^{2}=1 \text {. }
$$

i.e., either

or

$$
x_{1}^{2}+x_{2}^{2}+x_{3}^{2}+x_{4}^{2}+x_{5}^{2}-1=-2 \sin \lambda\left(x_{1} x_{5}+x_{2} x_{4}\right)-2 \cos \lambda\left(x_{1} x_{4}-x_{2} x_{5}\right),
$$

$$
x_{1}^{2}+x_{2}^{2}+x_{3}^{2}+x_{4}^{2}+x_{5}^{2}-1=2 \sin \lambda\left(x_{1} x_{5}-x_{2} x_{4}\right)-2 \cos \lambda\left(x_{1} x_{4}+x_{2} x_{5}\right) .
$$

If (1) occurs and there exists $\mathbf{y}_{1}=\left(y_{11}, y_{12}, y_{13}, y_{14}, y_{15}, 0\right)^{\prime}$ in ext $S(X)$ such that at least one of $y_{11} y_{15}+y_{12} y_{14}$ or $y_{11} y_{14}-y_{12} y_{15}$ is non-zero, then $\lambda$ can take at most two values in $[0,2 \pi]$. Consequently, the generators of the cylinders $\left\{C_{\beta}\right\}_{\beta \in B}$ arising from $\left\{X_{\beta}^{*}\right\}_{\beta \in B}$ contain at least one of four 2-dimensional subspaces. Hence the extreme points ext $S\left(X^{*}\right)$ of $S\left(X^{*}\right)$ lie in the union of at most four 4-dimensional subspaces. So the cylinders arising from $\left\{X_{\alpha}\right\}_{\alpha \in A}$ give rise to faces of $S\left(X^{*}\right)$ whose extreme points are (almost always) disconnected. So these faces cannot (almost always) be ellipses, which gives a contradiction.

So, if (1) occurs, then, for all extreme points in ext $S(X)$,

$$
x_{1} x_{5}+x_{2} x_{4}=0, \quad x_{1} x_{4}-x_{2} x_{5}=0
$$

and, if (2) occurs,

$$
x_{1} x_{5}-x_{2} x_{4}=0, \quad x_{1} x_{4}+x_{2} x_{5}=0 .
$$

We deal only with the case when (1), and hence (3), occurs; the argument when (2), and hence (4), occurs is similar.

From (3) we obtain

$$
\left(x_{1}^{2}+x_{2}^{2}\right) x_{5}=0 .
$$

Hence either $x_{1}=x_{2}=0$ or $x_{5}=0$. If $x_{1} \neq 0$ and $x_{5}=0$, then $x_{4}=0$. If $x_{2} \neq 0$ and $x_{5}=0$, then $x_{4}=0$. Consequently, either $x_{1}=x_{2}=0$, or $x_{4}=x_{5}=0$. So, if $X_{\alpha}$ is a 3-sphere amongst $\left\{X_{\alpha}\right\}_{\alpha \in A}$, but different from $X_{1}$ and $X_{2}$, then $X_{\alpha}$ meets one of $X_{1}, X_{2}$ in a 2 -sphere and we are again in case (i), which completes the proof of Lemma 8.

Combining Lemmas 7 and 8 and Proposition 4.4 of [1] (which says that if $\operatorname{dim} X=n$, 
$k(X) \neq n-1$ or $n-2)$ we obtain

THEOREM 3. Let $X$ be a Banach space of dimension at most six. Then $X$ has properties (1) to (3) of Theorem 1 only if one of the following conditions holds:

(i) $X$ is an inner product space;

(ii) $S(X)$ is a polytope with the property that for every facet $K$ of $S(X), S(X)$ is the convex hull of $K \cup-K$.

\section{REFERENCE}

1. J. Lindenstrauss and M. A. Perles, On extreme operators in finite dimensional spaces, Duke Math. J. 36 (1969), 301-314.

Dept. of Mathematics

UNIVERSITY COLLEGE LONDON

Gower Street, LoNDON WC1E 6BT 